\title{
Cognitive Design of an Application Enabling Remote Bases to Receive Unmanned Helicopter Resupply
}

\author{
Cynthia Dominguez, The MITRE Corporation; \\ Robert Strouse, Nationwide Children's Hospital; \\ Elizabeth Lerner Papautsky, Applied Research \\ Associates; \\ Brian Moon, Perigean Technologies
}

This paper reports on a research project that combined cognitive task analysis (CTA) methods with innovative design processes to develop a handheld device application enabling a non-aviator to interact with a highly autonomous resupply helicopter. In recent military operations, unmanned helicopters have been used to resupply U.S. Marines at remote forward operating bases (FOBs) and combat outposts (COPs). This use of unmanned systems saves lives by eliminating the need to drive through high-risk areas for routine resupply. The U.S. Navy is investing in research to improve the autonomy of these systems and the design of interfaces to enable a non-aviator Marine to safely and successfully interact with an incoming resupply helicopter using a simple, intuitive handheld device application. In this research, we collected data from multiple stakeholders to develop requirements, use cases, and design storyboards that have been implemented and demonstrated during flight tests in early 2014.

Keywords: Unmanned, helicopter, handheld device, interface design, human-robot interaction, cognitive task analysis, user experience

\section{Introduction}

The Autonomous Aerial Cargo/Utility System (AACUS) is an Innovative Naval Prototype program sponsored by the U.S. Office of Naval Research (ONR). The AACUS program is exploring advanced autonomous capabilities for reliable resupply to remote areas and, in the long term, casualty evacuation by an unmanned air vehicle under adverse conditions. Key features of AACUS include equipping a rotorcraft to autonomously avoid obstacles, to find - and land at - an unprepared site in dynamic conditions and to receive landing direction from a military field operator who is not an aviator. Due to an open architecture approach for global management of mission planning data, AACUS technologies will be platform agnostic and transferrable to both new and legacy cargo unmanned aerial systems (CUASs). When fielded, AACUS-enabled rotorcraft will ideally be able to rapidly respond to requests for support, operate in all weather conditions, be launched from sea and land, fly in high and hot environments, and autonomously detect and negotiate landing sites in potentially hostile settings. Such missions could require

\footnotetext{
Authors retain copyright and grant the Journal of Human-Robot Interaction right of first publication with the work simultaneously licensed under a Creative Commons Attribution License that allows others to share the work with an acknowledgement of the work's authorship and initial publication in this journal.
}

Journal of Human-Robot Interaction, Vol. 4, No. 2, 2015, Pages 50-60, DOI 10.5898/JHRI.4.2.Dominguez 
significant obstacle and threat avoidance, with aggressive maneuvering in the descent-to-land phase (ONR, 2013).

Our involvement in the AACUS program is in support of a team led by Aurora Flight Sciences, who has outfitted a Boeing Unmanned Little Bird helicopter with perception, control, and planning technologies to create an AACUS-enabled system (AES). Our primary goal has been to provide the team with a human-centered design of interfaces for interacting with the autonomous air vehicle. Our design process initiated with a cognitive task analysis (CTA), followed by a series of design workshop and validation activities.

This design process was geared toward understanding and supporting the envisioned world of a non-aviator Marine at a combat outpost (COP) who is responsible for interacting with the AES to accomplish missions of resupply and casualty evacuation (CASEVAC). The term "envisioned world" is used when designing a system that does not exist in today's operational environments (Dekker \& Woods, 1997). Parts of this AACUS scenario have been carried out in operations in Afghanistan with the K-MAX demonstrator system (Lockheed Martin, 2013) and through manned helicopter operations. However, the AACUS program represents several first-ofa-kind departures from today's operations. First, the AES is envisioned to be supervised for landing operations by a Marine at the combat outpost who has not had formal training in UAS or flight operations. In Afghanistan, the K-MAX helicopter has been flown by well-trained UAS operators into prepared landing sites. (A primary aviation-trained operator at a main operating base would remain responsible for the aircraft.) Second, the AACUS aircraft will be capable of carrying internal loads, whereas K-MAX carries its cargo via a sling load. Finally, and perhaps most significantly, the AACUS-enabled helicopter will employ highly automated perception/planning systems, with which the main operating base (MOB) primary operator and the COP field operator will be required to understand and interact. Due to the complexities and multiple areas of expertise involved in remote UAS operations, design of interfaces for intuitive use by a Marine field operator requires principled understanding of the operational expertise of a range of subject-matter experts (SMEs).

This paper captures the process of how we researched, developed, and created app functionality and designs for the AACUS COP field operator (FO). We begin with an explanation of cognitive task analysis and how we used it to understand the COP FO's envisioned world and create context-relevant, intuitive designs. We will detail interim steps in this process, including development of requirements and use cases; those processes and products will be described and examples provided. Finally, we will describe the design process and the validation methods used during the AACUS Phase I project.

The sequence of the paper reflects the general order of the work we performed. However, the iterative, discovery-based nature of CTA work influenced the process, and can be seen in the way that we revisited requirements and use cases after design was well under way.

\section{CTA Process}

CTA is a family of data collection and analysis tools developed and used to identify and describe the elements that underlie skilled cognitive performance (Crandall, Klein, \& Hoffman, 2006). Through the use of incident accounts, case histories, cognitively authentic simulations, and observations, this family of methods enables researchers and developers to elicit detailed descriptions of what people attend to, how they make sense of complex situations, and how they use information in the course of making decisions.

Learning about a complex, high-threat environment, such as a COP, requires an iterative approach, because in these situations it is typically not clear what the important knowledge is until you start to gather data. We began by conducting interviews with personnel from our company (ARA) who were retired helicopter pilots and retired Army Special Operations Forces (SOF) (we interviewed three of each). In these first interviews, we asked a range of general knowledge 
questions about resupply and helicopter support to understand how units in remote environments communicate with, and interact with, rotorcraft and the military logistics support.

These early pilot interviews gave us an initial understanding of the operational and organizational context that our target operator, a Marine at a combat outpost, will experience when conducting resupply and CASEVAC. Following this initial set of interviews, we parsed the expertise needed into the following areas:

1. How do ground troops organize and act within their military systems and environments to receive resupply and conduct CASEVAC operations?

2. How do military helicopter pilots organize and act within their systems and environments to deliver resupply and conduct CASEVAC operations?

3. What are the unique characteristics and constraints of combat outposts (COPs) and forward operating bases (FOBs) that have impact on the future operations of an AACUS enabled system?

4. How do Marines currently operate unmanned systems in both resupply (K-MAX demonstrator) and ISR (intelligence, surveillance, and reconnaissance) missions?

Armed with the retirees' initial understanding, we planned two data collection trips. During the first, we went to the Marine Corps Air Ground Combat Center in Twentynine Palms, CA, to interview Marine UAS pilots who had flown either K-MAX or other UAS systems. The second trip was to The Basic School in Quantico, VA, to interview the school's Marine instructors who had either pilot or ground-based CASEVAC and resupply experience, with the majority having $\mathrm{COP}, \mathrm{FOB}$, or MOB experience as well. We conducted a total of $23 \mathrm{CTA}$ interviews before developing requirements, use cases, and finally storyboard designs via a design workshop. Another nine validation interviews were then accomplished with the initial set of storyboards. This range of expert perspectives allowed us to triangulate on a representative understanding of the envisioned world problem of AACUS. Triangulation refers to integrating the various perspectives of these experts, looking for replication and differences in the data, and thereby understanding the complexities and interactions in the future operating system/environment. Fig. 1 represents the subject matter expertise we captured to triangulate an understanding of the COP FO's task and operational environment.

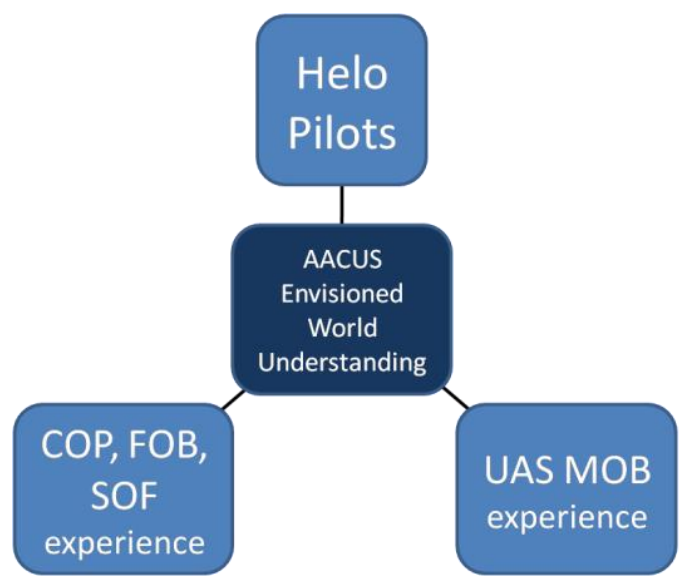

Figure 1. Triangulated representation of the COP FO's task and operational environment

Specifically, during the Twentynine Palms data collection, we focused on understanding, in detail, issues likely to affect the interface design during Approach, Imaging, and Landing phases. For example, we examined wind data (speed, direction, variability) considerations and 
contingency planning, since these would be most revealing of the field operator's tasks in difficult situations. In addition, with some of the participants, we conducted simulation interviews addressing resupply and CASEVAC missions to explore wave-off and designation of a new landing zone (LZ) in detail, as well as the interface requirements (e.g., fuel status, ETA) to support these activities. We gathered multiple examples of contingency planning as well as critical incidents, including fuel contingencies due to wind. At Quantico, we were able to interview Marine officers who had been involved in resupply and CASEVAC at COPs and FOBs, both from the air and from the ground. These interviews enabled us to capture the in-depth descriptions of these operating locations and their constraints. We also captured information at Quantico on the CASEVAC mission that included contingency planning, timing considerations, and system trust. These data collection events served as a rich source of information for our data analysis products. Table 1 summarizes all of the project's data collection and validation events, including background of participants, number of participants, location and date of interviews, focus of interview, and analysis products that were informed by the findings.

Table 1. Summary of Knowledge Elicitation and Design Validation Data Collection Opportunities

\begin{tabular}{|c|c|c|c|c|}
\hline Task & $\begin{array}{l}\text { Background of } \\
\text { Participants }\end{array}$ & $\begin{array}{l}\text { Number of } \\
\text { Participants }\end{array}$ & Focus of Interview & $\begin{array}{l}\text { Analysis } \\
\text { Products }\end{array}$ \\
\hline $\begin{array}{l}\text { CTA Round } \\
1\end{array}$ & $\begin{array}{c}\text { Tier } 1 \text { Army Special } \\
\text { Operations Forces } \\
\text { retirees }\end{array}$ & 3 & Resupply; insertion; extraction & $\begin{array}{c}\text { Diagram } \\
\text { representation; } \\
\text { CTA-driven HSI } \\
\text { requirements } \\
\end{array}$ \\
\hline $\begin{array}{l}\text { CTA Round } \\
1\end{array}$ & $\begin{array}{l}\text { Retired helicopter } \\
\text { pilots }\end{array}$ & 3 & $\begin{array}{l}\text { Helicopter pilot perspective for } \\
\text { insertion and extraction }\end{array}$ & $\begin{array}{c}\text { Diagram } \\
\text { representation; } \\
\text { CTA-driven HSI } \\
\text { requirements } \\
\end{array}$ \\
\hline $\begin{array}{l}\text { CTA Round } \\
\text { 2: COP } \\
\text { Interface } \\
\text { Design } \\
\end{array}$ & $\begin{array}{l}\text { Marine KMAX and } \\
\text { other UAS operators }\end{array}$ & 12 & $\begin{array}{l}\text { Approach/Imaging/ } \\
\text { Landing, wind data } \\
\text { considerations, and } \\
\text { contingency planning }\end{array}$ & $\begin{array}{l}\text { CTA-driven HSI } \\
\text { requirements; use } \\
\text { cases; storyboards }\end{array}$ \\
\hline $\begin{array}{l}\text { CTA Round } \\
\text { 2: COP } \\
\text { Interface } \\
\text { Design } \\
\end{array}$ & $\begin{array}{l}\text { Marine instructors at } \\
\text { The Basic School }\end{array}$ & 4 & $\begin{array}{l}\text { Resupply and CASEVAC } \\
\text { missions to include } \\
\text { contingency planning, timing } \\
\text { considerations, and system trust }\end{array}$ & $\begin{array}{l}\text { CTA-driven HSI } \\
\text { requirements; use } \\
\text { cases; storyboards }\end{array}$ \\
\hline Validation & $\begin{array}{l}\text { Marine KMAX and } \\
\text { UAS operator }\end{array}$ & 1 & Validate COP design concepts & Design updates \\
\hline Validation & $\begin{array}{l}\text { Air Guard security } \\
\text { police }\end{array}$ & 9 & Validate COP design concepts & Design updates \\
\hline Validation & $\begin{array}{l}\text { Current Marine } \\
\text { officers and former } \\
\text { Marine logistics } \\
\text { officer }\end{array}$ & 3 & Validate COP design concepts & Design updates \\
\hline Total & & 35 & & \\
\hline
\end{tabular}

Individual interviews were conducted by two experienced interviewers and lasted from one to two hours. We asked questions about participants' background, including age, rank/position, years of experience, deployments, and relevant operational experience (dependent upon interview focus). Following an interview guide that was developed for each data collection event based on interview focus and participant background, we asked interview questions on a series of predetermined core topics, such as key tasks/responsibilities in conducting various types of missions, operational conditions, contingency planning, operator challenges, and technical risks. We elicited information about the environments in which AACUS will be used, the challenges associated with operating manned and unmanned rotorcraft, the cues to which they attended during these missions, and other cognitive factors associated with the future AACUS mission and operating environments. We used CTA methods such as the task diagram (Hutton \& Millitello, 1996) to 
understand procedural tasks involved in flying and resupply and the critical decision method (Crandall \& Getchell-Reiter, 1993) to elicit context-rich lived incidents. We also used the simulation interview method (Hutton \& Millitello, 1996) in which participants were asked to roleplay as a Marine logistics specialist at a COP, interacting with an autonomous helicopter providing resupply, as we walked them into a detailed scenario with contingencies and 'what-ifs.'

\section{CTA Analysis and Products}

The analysis of this large set of text-based data was iterative and theme-based. To start, we developed a detailed set of interview notes and audio recordings for each interview. We analyzed interview data using qualitative analysis techniques, assigning team members to do multiple data passes in a structured thematic analysis, identifying common themes and patterns in participant responses. Specific attention was paid to job and task performance factors that would either facilitate or hinder effective interaction with the AACUS system.

We created a set of analysis products based on the CTA interviews that included requirements, use cases, and COP HSI storyboards. As an initial step to understanding the operational domain, we also created a concept map representation based on the early interviews we conducted with ARA retired SOF personnel and helicopter pilots.

\subsection{Concept Map Development}

To develop this concept map of critical tasks, we systematically pulled from interview notes the tasks associated with each mission phase and clustered them according to the order of execution. We put data from ground-based perspective below the timeline, in olive, and data from helicopter pilot participants above the timeline in blue. Additionally, we noted instances in which tasks were identified as being particularly challenging. The representation served as a framework for understanding helicopter mission phases for CASEVAC and resupply missions. This diagram is too large to include here, but it was extremely helpful to map early data with the mission and to share the nature of the data and analysis with developers and sponsors as it evolved.

\subsection{CTA-Driven HSI Requirements Development}

We used all sources of CTA data to inform HSI requirements, starting with the in-house ARA interviews. For each set of interview notes, we systematically identified and listed requirements identified by SMEs, either when discussing their needs as part of giving an example, recounting an incident, or in response to an explicit prompt. We used a collaborative and iterative approach to requirements development, with team members nominating, eliciting feedback on, and revising requirements accordingly, based on interview data. Working with the larger AACUS team, and most closely with the application developers, we created and socialized a set of interface design requirements that directly reflected interview data.

A subset of the entire requirements list was designated as relevant to AACUS Program Phase I (i.e., requirements that became the basis for AACUS Phase I design). Thinking toward the future, requirements that will not be implemented in Phase I, due to the scope of the Phase I demonstration, were also assembled and are being tracked going forward.

To ensure capture of material that was generated during and after the design workshop, an additional sweep through all of the CTA interviews was conducted. ARA team members flagged information that was not included in current requirements, but which might be, and wrote draft requirements. These new requirements also reflected design directions or decisions made during the workshop and subsequent storyboard development.

Requirements were categorized into a structure that enabled us to communicate them more clearly across the larger team, and to easily apply them to design questions. Example categories were mission planning, situation awareness, dynamic replanning, and landing zone operations. Sample requirements are shown in Table 2. 
Dominguez et al., Cognitive Design of Handheld UAS App

Table 2. A Sample of Requirements Categories and Requirements

\begin{tabular}{|r|}
\hline 1. Mission Planning \\
\hline 1. \\
$\begin{array}{l}\text { The COP and MOB interfaces shall integrate with the appropriate air tasking and } \\
\text { planning systems, and indicate airspace and landing zone conflicts. }\end{array}$ \\
\hline 2. Situation Awareness \\
\hline $\begin{array}{l}\text { The system shall indicate intended and completed actions via the COP interface. } \\
\text { Intended actions include: ingress and egress directions, changes in heading, airspeed, } \\
\text { and altitude, next executions, approvals, and landing information. }\end{array}$ \\
\hline 2. $\begin{array}{l}\text { The COP interface shall enable the user to visualize and follow the Mission Plan as it is } \\
\text { carried out. }\end{array}$ \\
\hline 3. $\begin{array}{l}\text { The system shall compare its present and historic states to previous states and conditions } \\
\text { to determine whether it is operating within acceptable tolerances. If it is operating } \\
\text { outside tolerances, the system should indicate specific violations and suggested } \\
\text { responses to the COP and MOB operator interfaces. }\end{array}$ \\
\hline 3. Dynamic Replanning \\
\hline 1. $\begin{array}{l}\text { The system shall receive and execute revised Mission Plans while in-flight, from the } \\
\text { COP and MOB interfaces, without interruption of flight. }\end{array}$ \\
\hline 2. $\quad$ The system shall be abort capable at any time. \\
\hline 3. $\begin{array}{l}\text { The system shall provide an engine kill switch for use when the aircraft is on the } \\
\text { ground. }\end{array}$ \\
\hline
\end{tabular}

\subsection{Use Case Development}

Using data from all of our CTA interviews, we developed use cases that elaborate on the seven program scenarios (sorties) provided by ONR. The cases build from a benign baseline scenario into various branches and sequels to describe the unique situations presented by each sortie. In summary, the scenarios were:

1. Baseline demonstration: aircraft flies an approach, selects a safe landing site, lands, and takes off without incident;

2. Baseline plus obstacle avoidance in flight;

3. Baseline plus field operator abort/wave off (early in approach to landing zone);

4. Baseline plus field operator abort/wave off (late in approach to landing zone);

5. Baseline plus redirect to an alternate landing point due to aircraft judging the designated landing site to be infeasible;

6. Baseline plus field operator provides additional context regarding a ground constraint or obstacle; and

7. Baseline plus loss of communications with the COP field operator.

We next created a Use Case Architecture to serve as a roadmap for further use case development. The Use Case Architecture aligned the mission timeline-based scenarios with key findings from our CTA data and called out responsible parties for each task or decision point. Using this use case architecture as a roadmap, we developed a one-page document for each of the elements of the architecture. The CTA data helped our team add detail to the tasks and decision points and to compose descriptive answers to three questions for each element, which were: "What is going on in the World?" "What is going on with the Field Operator?" and, "What is going on with the System?" 


\subsection{Design Workshop}

The initial COP HSI design concepts were generated during a COP Design Workshop. The workshop attendees consisted of our immediate team, our collaborating software developers, and our AACUS Program Manager from Aurora Flight Sciences. Our resident designer (co-author Robert Strouse) planned and facilitated the workshop. (Ideally, one or more Marines with logistics experience would have participated, but none were able to attend.)

The use cases and requirements were provided to workshop participants and were used as a key input to designs. Participants were also asked to come to the workshop prepared to share two mobile apps with the group - one that they found interesting, and one that someone who was unlike themselves found interesting. The opening exercise of sharing these mobile apps and explaining why they are interesting created a baseline of the possibility for creating our own mobile app for AACUS.

With our data analysis products and the ONR mission scenarios in mind, we created a list of key 'How Might We...' questions that, if answered, would provide us with the necessary insights to inspire design work. The final list of these questions is shown in Table 3.

Table 3. List of 'How Might We...'

brainstorming questions for the COP HSI handheld display

\begin{tabular}{|l|}
\hline \multicolumn{1}{|c|}{ HOW MIGHT WE... } \\
\hline Display aircraft health/status parameters on handheld device? \\
\hline Negotiate a landing with the aircraft through a handheld device? \\
\hline $\begin{array}{l}\text { Negotiate a successful landing when the operator informs the } \\
\text { system of a threat at the current LZ? }\end{array}$ \\
\hline $\begin{array}{l}\text { Display information regarding LZ and touchdown zone (TZ) and } \\
\text { enable selecting an LZ? }\end{array}$ \\
\hline Communicate known obstacles near the LZ to the aircraft? \\
\hline Display 'who has control' information? \\
\hline Push information that represents a deviation from the flight plan? \\
\hline Display route information? \\
\hline Display and select landing profile options? \\
\hline
\end{tabular}

During the design workshop, we used the "How might we..." questions to methodically explore what a COP HSI interface could display on its screen to allow an untrained Marine to successfully monitor and interact with the autonomous vehicle. Large group ideation sessions were followed by smaller group design sessions, during which team members created low-fidelity paper prototypes. Each paper prototype was then presented to the group; the presentation was videotaped to capture the rationale. Fig. 2 shows some of the sketches created.

\subsection{Storyboard and Interface Design Development}

By the end of the workshop, the team had prototyped several concepts for each COP HSI component. The best prototype from each component group was selected by assessing its ability to 

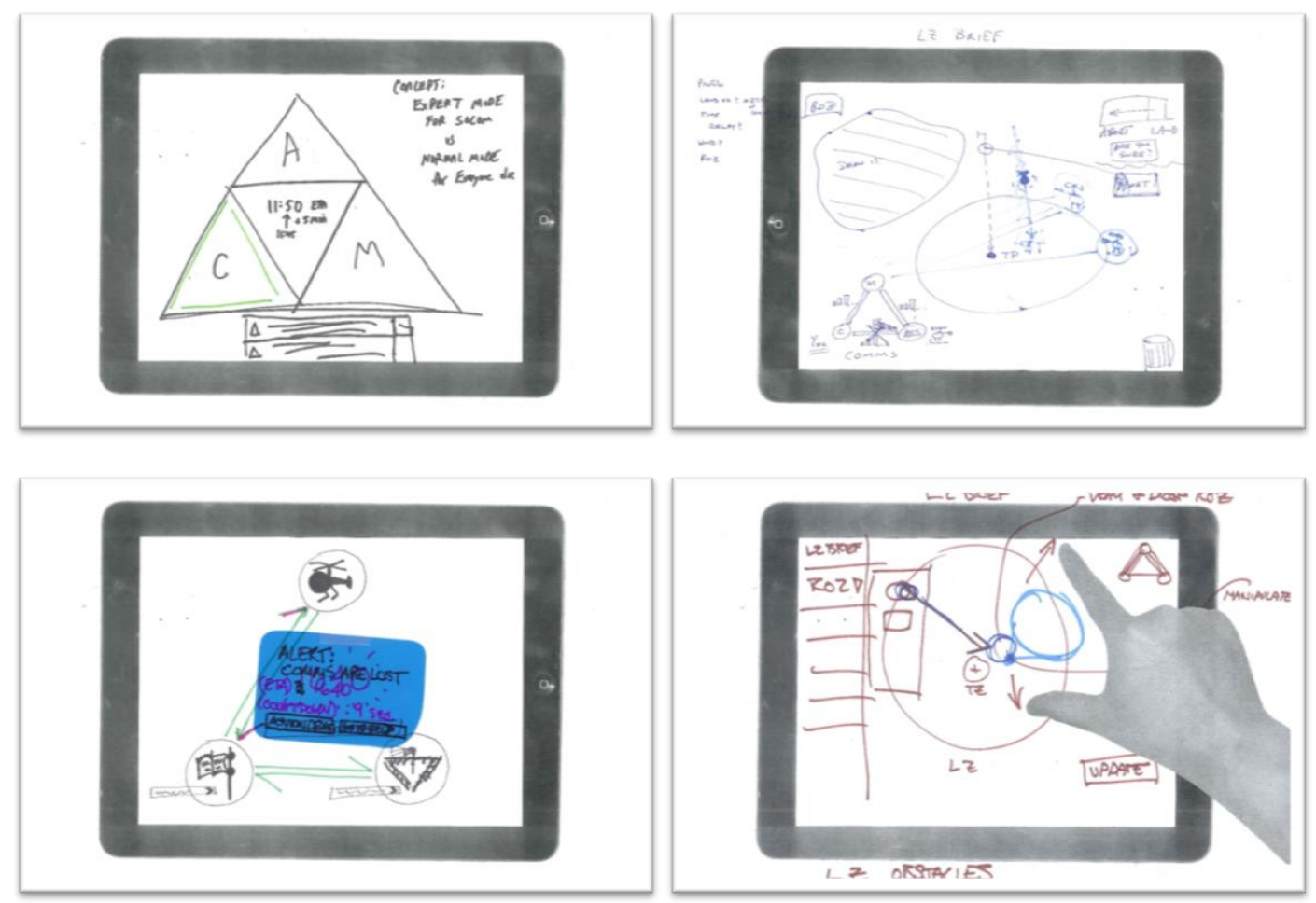

Figure 2. Sketches created by small design teams following problem-oriented ideation sessions

enable the untrained Marine operator to perform operational tasks, with graphical simplicity becoming the differentiator between competing concepts. These concepts were merged and developed in a format that emulated full-size imagery and text on an iPad mini, which was selected as the operational best of breed tablet.

Using the Use Case Architecture, we then created a storyboard for each use case element. Each storyboard addressed an ONR scenario and included a comic-book-like cell depicting the untrained Marine in a forward position, a short written description of what is happening, and a screenshot of what the COP HSI would look like in that exact moment. An example of these storyboards can be seen in Fig. 3.

\section{Validation}

Design products such as these require validation, being early paper products and essentially hypotheses for how the interface meets the requirements we had developed. We conducted validation with a Marine officer and UAS pilot who had hosted us at Twentynine Palms, and then we conducted further formal testing with Air Guard security police at a local National Guard facility. Air Guard security forces were an accessible military population who agreed to volunteer and who shared background characteristics and experience with our intended Marine user. 


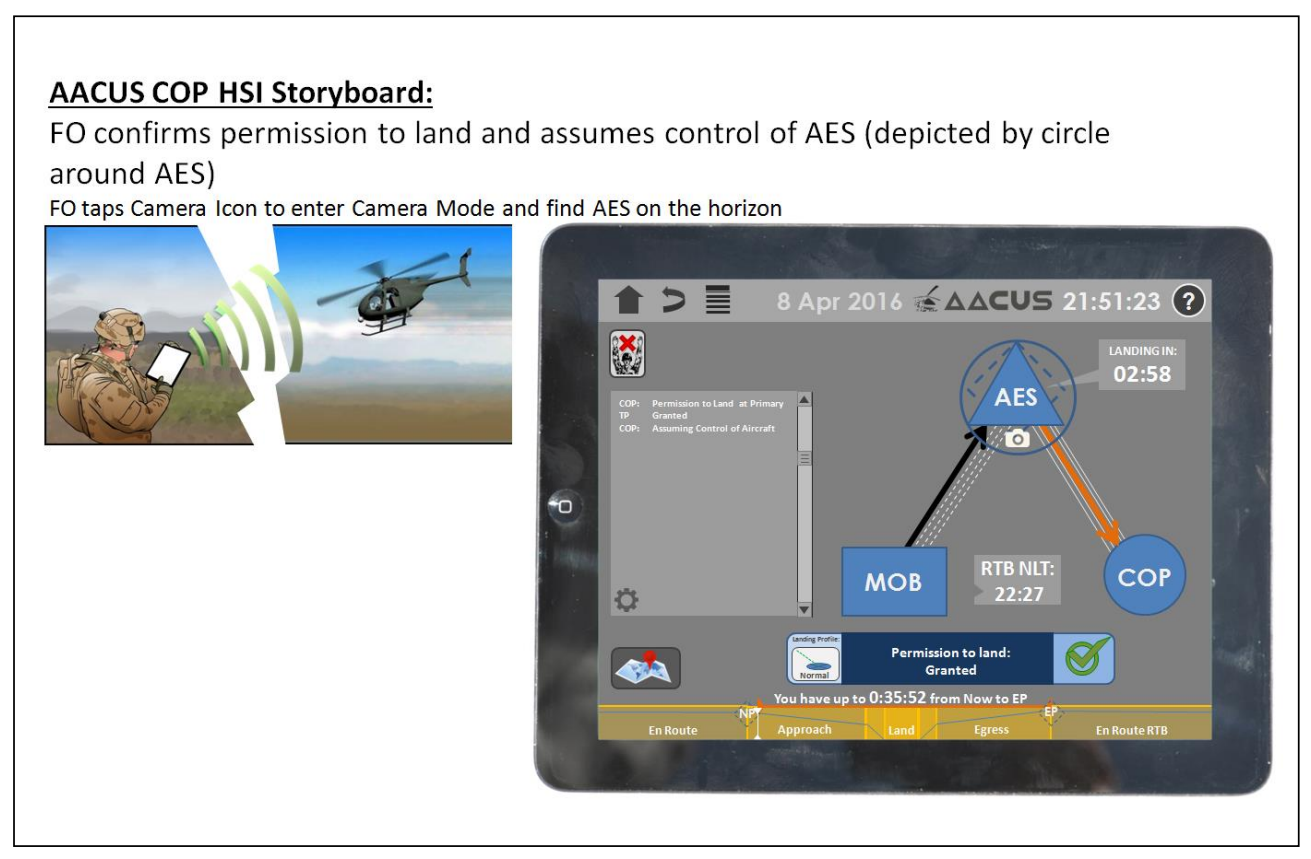

Figure 3. Initial COP HSI Storyboard

The validation testing was intended to meet multiple goals. Those goals included:

1. Evaluate the effectiveness of a 15-minute orientation to the COP HSI to gauge the feasibility of introducing an untrained user to the interfaces (a self-imposed technical performance measure, or TPM, of our design was that a Marine with no more than 15 minutes of training could operate the handheld interface);

2. Determine the best option for supporting the field operator's time-critical "Infeasible Touchdown Zone" negotiation, for which we had designed three options;

3. Identify components for re-design or additional training; and

4. Seek design improvements and validate the overall interface.

To address these goals, we developed and followed a procedure that included an orientation session, administration of a 13-question test designed to assess the technical performance measure (TPM), and a validation and usability evaluation. A training session was a 15-minute orientation to the handheld interface, which was shown in static images on an iPad mini. The TPM test asked questions about how participants would interact with the interface across a variety of scenarios (e.g., point to/push/simulate appropriate action on the static image). We administered the TPM questions both before and after we provided the training to assess how intuitive the screens were by themselves without orientation. We measured participants' accuracy of response (correct/incorrect) in addition to participants' time to completion.

Validation testing demonstrated the feasibility of the brief orientation session. More importantly, the testing provided valuable feedback on specific design questions we had, and ideas for redesigns, which the participants generated from the testing experience. We folded the feedback into our designs to create a final, validated set of COP HSI storyboards, an example of which is shown in Fig. 4. 


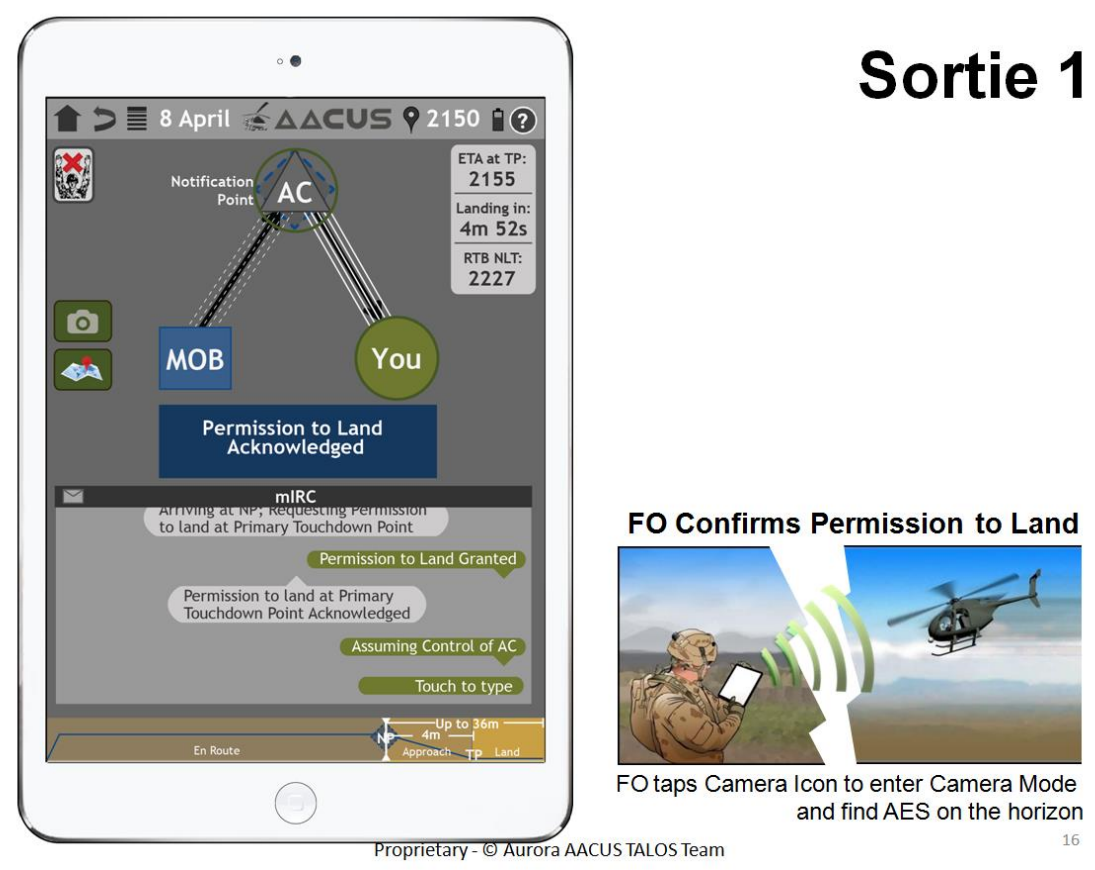

Figure 4. Example of a final, validated COP HSI storyboard

\section{Conclusion}

This paper captures a combination of cognitive task analysis and collaborative design methods applied to the design of handheld interfaces for a first-of-a-kind military system. A critical component of the success of this effort was the participation of software engineers and program management in our interviews and design workshops. Design storyboards were familiar and wellunderstood when provided to the software engineering team, since these engineers had been a part of interviews and design workshops. The entire AACUS-enabled system, to include our COP app, was tested in a live flight demonstration and evaluation in early 2014. During that demonstration, the Marine Lance Corporal who used the app to interact with the AACUS-enabled helicopter judged the interface to be extremely easy to operate; he was given just 15 minutes of training and some extended field coaching by our development team. We gathered additional feedback from observing his use of the interface and from his comments and insights.

We focused our design efforts on the envisioned world of an untrained Marine receiving an unmanned helicopter resupply. Hopefully this process provides a valuable case study for the design of mobile interfaces to support human-robotic interaction. Our approach provides a roadmap and examples of artifacts that may guide future design efforts.

\section{Acknowledgements}

The authors wish to acknowledge the sponsorship of ONR for this effort, the guidance and vision of ONR program manager Missy Cummings to require cognitive analysis for this program, and the leadership of Aurora Flight Sciences, particularly Program Manager John Wissler and Principal Investigator James Paduano. We would also like to acknowledge our collaborators at Kutta 
Technologies Inc., particularly James Bona, for his guidance in software engineering and supportive design participation, and Desare'a Chessar, for her instrumental role in support of validation and design efforts. Most importantly, we wish to thank the 35 participants who supported our CTA and design testing activities, whose future challenges we hope will be aided by our efforts.

\section{References}

Crandall, B., \& Getchell-Reiter, K. (1993). Critical decision method: A technique for eliciting concrete assessment indicators from the "intuition" of NICU nurses. Advances in Nursing Sciences, 16(1), 42-51.

Crandall, B., Klein, G., \& Hoffman, R. R. (2006). Working minds: A practitioner's guide to Cognitive Task Analysis. Cambridge, MA: The MIT Press.

Dekker, S., \& Woods, D. (1997). The envisioned world problem in cognitive task analysis. In Proceedings of the ONR/NATO Conference on Cognitive Task Analysis. Washington, D.C.

Hutton, R. J. B., \& Millitello, L. G. (1996). Applied Cognitive Task Analysis (ACTA): A practitioner's window into skilled decision making. In D. Harris (Ed.), Engineering psychology and cognitive ergonomics: Job design and product design (Vol. 2, pp. 17-23). Aldershot, UK: Ashgate.

Lockheed Martin. (2013). K-MAX. Available at http://www.lockheedmartin.com/us/products/ kmax.html

ONR (2013). Autonomous Aerial Cargo/Utility System Program Description. Available online at http://www.onr.navy.mil/Science-Technology/Departments/Code-35/AllPrograms/aerospace-research-351/Autonomous-Aerial-Cargo-Utility-AACUS.aspx

Authors' names and contact information: Cynthia Dominguez, PhD, The MITRE Corporation, Bedford, MA, USA. Email: cinguez234@gmail.com; Robert Strouse, MFA, Nationwide Children's Hospital, Columbus OH, USA. Email: robert.strouse@gmail.com; Elizabeth Lerner Papautsky, PhD, Applied Research Associates, Cognitive Solutions Division, Fairborn, OH, USA. Email: lpapautsky@ara.com; Brian Moon, Perigean Technologies LLC, Fairfax, VA, USA. Email: brian@perigeantechnologies.com.

The first author's affiliation with The MITRE Corporation is provided for identification purposes only, and is not intended to convey or imply MITRE's concurrence with, or support for, the positions, opinions or viewpoints expressed by the author. This paper has been approved for public release by the Office of Naval Research. 